\title{
Theoretical performance of serrated external occulters for solar coronagraphy
}

\section{Application to ASPIICS}

\author{
R. Rougeot ${ }^{1,2,3}$ and C. Aime ${ }^{1}$ \\ ${ }^{1}$ Université Côte d'Azur, Observatoire de la Côte d'Azur, CNRS UMR 7293, Lagrange, Parc Valrose, 06108 Nice Cedex 2, France \\ e-mail: raphael.rougeot@esa.int \\ ${ }^{2}$ European Space Research and Technology Center, European Space Agency, Keplerlaan 1, 2201 Noordwijk, The Netherlands \\ 3 Thales Alenia Space, 5 allée des Gabians, 06150 Cannes, France
}

Received 19 December 2017 / Accepted 17 January 2018

\begin{abstract}
Context. This study is made in the context of the future solar coronagraph ASPIICS of the ESA formation-flying mission Proba-3. Aims. In the context of solar coronagraphy, we provide a comparative study of the theoretical performance of serrated (or toothed) external occulters by varying the number and size of the teeth, which we compare to the sharp-edged and apodized disks. The tooth height is small (a few centimeters), to avoid hindering the observation of the solar corona near the limb. We first analyze the diffraction pattern produced by such occulters. In a second step, we compute the umbra profile by integration over the Sun.

Methods. We explored a few methods to compute the diffraction pattern. Two of them were implemented. The first is based on 2D fast Fourier transformation (FFT) routines and a multiplication by the Fresnel filter of the form $\exp \left(-\mathrm{i} \pi \lambda z u^{2}\right)$. Simple rules were derived and discussed to set the sampling conditions. The Maggi-Rubinowicz representation is then proposed as an alternative method, and is proven to be very efficient for this study.

Results. Serrated occulters tend to create a two-level intensity pattern, the inner being the darker, which perfectly matches a previously reported geometrical prediction. The diffraction in this central region is lower by two to four orders of magnitude when compared to the sharp-edged disk. The achieved umbra level at the center ranges from $10^{-4}$ to below $10^{-7}$, depending on the geometry of the teeth. Conclusions. Our study shows that serrated occulters can achieve a high rejection and can almost reach the performance of the apodized disk when very many teeth are used. We prove that shaped occulters must be preferred to simple disks in solar and stellar coronagraphy.
\end{abstract}

Key words. Sun: corona - instrumentation: high angular resolution - methods: numerical

\section{Introduction}

The first experiment using an external occultation was made by Evans (1948) to measure the brightness of the sky near the Sun. On a highly reduced scale, this experiment simulates the phenomenon of a lunar eclipse and has been the precursor of a series of coronagraphs using an external occulter in space, as was well described by Koutchmy (1988) in his review of space-borne coronagraphy. The principle of these experiments appears to be much simpler than the classical Lyot coronagraph (Lyot 1939), but characterizing the straylight involves Fresnel diffraction, the effects of which are very complex to model accurately.

Very soon after the first experiments in the 1969s, it was considered that a single perfect circular disk was not the best external occulter to observe the solar corona. Following the concept of apodization in optics as formalized by Jacquinot \& Roizen-Dossier (1964), apodized or shaped occulters were imagined. They are intended to provide a shadow much darker than the disk. Multiple disks or serrated occulters were thus tried out, as described in Newkirk \& Bohlin (1965) or Purcell \& Koomen (1962), for example. The intuitive reasoning of the latter authors to use a toothed disk was that it this disk would diffract the incoming light waves in a direction perpendicular to the edge. Therefore, a serrated mask made of successive teeth was thought to reject the light outside a central part, creating a strong darkness. Conversely, a simple disk would reject the light toward the center, resulting in an inferior good darkness. Based on this reasoning, Boivin (1978) deduced by geometrical considerations that such a serrated occulter would produce a dark inner region whose radius, which we name the Boivin radius, depends on the number and the size of the teeth.

In stellar coronagraphy, it is planned to use shaped occulters for the direct detection of exoplanets, and they have been extensively investigated, which led to numerous publications in the past decade. Typically, the occulter is about $30 \mathrm{~m}$ in diameter, located at $\sim 80000 \mathrm{~km}$ from a $\sim 4 \mathrm{~m}$ telescope. The contrast to be achieved is $10^{-10}$. It has been proven that an optimized apodization function for occulter provides the best rejection performance. However, it is known that ideal radial apodization remains an ideal that cannot be manufactured with the required precision. As an alternative, Cash (2006) and Vanderbei et al. (2007) proposed and investigated the use of a petal-shaped occulter, which is an improved version of the serrated occulter made 
of petals of more elaborated shape compared to the mere triangular tooth envisaged in previous solar studies. Moreover, the adopted point of view is rather different from the geometrical reasoning discussed above for the solar case. Here, the petalized occulter is seen as a discrete substitute of the ideal radially apodized occulter, as described in Vanderbei et al. (2007).

Furthermore, the goals and therefore the geometry of solar and stellar coronagraphy are quite different. The relative closeness of observation from the bright source is not as severe for the exoplanet as it is for the solar corona, and the extended light source that is the Sun is not comparable to a stellar point-source. This implies that the diffraction pattern must be known to a large extent, and not simply in a very central part. We recall that the solar coronagraph ASPIICS is made of an occulter with a $1.42 \mathrm{~m}$ diameter positioned $144 \mathrm{~m}$ in front of a telescope that in turn has a $5 \mathrm{~cm}$ aperture (Lamy et al. 2010; Renotte et al. 2015). This Lyot coronagraph will fly on the future ESA formation-flying mission Proba-3, and is split between two spacecraft. Related modeling of Fresnel diffraction for the sharp-edged occulting disk has previously been studied in Aime (2013) and Rougeot et al. (2017), where the diffracted wavefront is propagated through the hybrid Lyot coronagraph.

Only a few studies about serrated occulters for solar coronagraphy can be found in the literature. Fort et al. (1978) illustrated the gain in straylight of a polished toothed circular disk by experimental measurements, which supports the early suggestion of Purcell and Koomen. In particular, they highlight the required quality of the edge of the occulter, and associated manufacture difficulties. In a more recent study, related to the ASPIICS solar coronagraph, Verroi et al. (2008) developed a diffraction modeling based on Fresnel-Kirchhoff theory and a clever splitting of the plane of the occulter in Fresnel zones. They computed the diffraction intensity for a toothed external occulter and reported a remarkable reduction of the amount of diffracted light at the entrance aperture. However, they considered only a few teeth, about 20, and the shape is different from the classic straight line that we investigate in this paper. Our results are therefore not comparable to their results. Finally, Landini et al. (2016) presented an experimental study of the rejection of various external occulters, based on a reduced scale test bench.

We present here a theoretical and numerical study of diffraction for serrated occulters, using the parameters of the long-base solar coronagraph ASPIICS. We aim to analyze the rejection performance of serrated occulters for solar coronagraphy by varying the number and the size of the teeth, and to compare a sharp-edged and an apodized disk. We report that a diffraction computation like this remains a very delicate operation, for instance, for the sampling constraints, and several models have been investigated and implemented for this problem. The penumbra profile is obtained by a mere convolution with the solar image. In particular, we give the trend of the integrated illumination over the $5 \mathrm{~cm}$ aperture of the ASPIICS telescope with respect to the number and the size of the teeth.

The paper is organized as follows. Section 1 contains this introduction. In Sect. 2 we introduce the different models we investigated to compute diffraction, and the related numerical implementation. In Sect. 3 we analyze the diffraction pattern obtained for serrated occulters. We show that our results are in good agreement with the intuitive reasoning of Boivin (1978). The computation of the penumbra profile is addressed and discussed in Sect. 4. Conclusions are given in Sect. 5.

\section{Diffraction by an external occulter}

\subsection{Fresnel integral}

The Fresnel diffraction of a thin external occulter that is assumed to be a flat structure lying in a plane can be obtained using classical Fourier optics, as described in Goodman (2005), for example. This theory is based on paraxial optics, which approximates a sphere by a paraboloid. This defines the Fresnel regime of diffraction, which has proved to be sufficiently accurate for optical astronomy.

In the following, we set a Cartesian coordinate system $(x, y, z)$. We associate a set of polar coordinates $(r, \theta)$ to the transversal plane $(x, y)$. The occulter is set in the plane $z=0$. In the Fourier optics formalism, all waves propagate in the $z$ positive direction, and amplitudes are described in the transversal planes $(x, y)$ for different $z$ values. For example, the propagation of a monochromatic plane wave of unit amplitude (corresponding to rays parallel to the $z$-axis) is represented in this scalar theory of light by the phase term $\exp (2 \mathrm{i} \pi z / \lambda)$, where $\lambda$ is the wavelength of the light and $z$ is the covered distance from the origin. A tilted plane wave propagating with small angles $(\alpha, \beta)$ with regard to the $z$-axis is affected, in addition to this, by a phase term $\exp (-2 \mathrm{i} \pi(\alpha x+\beta y) / \lambda)$. The tilted wave is still considered as propagating toward the $z$ direction in this paraxial optics theory, so that the interaction of coherent waves of different angles $(\alpha, \beta)$ can be considered. As an illustration, two such coherent waves of different angles produces Young fringes.

More generally, let $\Psi_{0}(x, y)$ be the complex amplitude of a wave in plane $z=0$ (including the occulter). Neglecting the irrelevant constant phase term $\exp (2 \mathrm{i} \pi z / \lambda)$ that is related to the propagation of a plane wave, the complex amplitude $\Psi_{z}(x, y)$ of the wave at the distance $z$ can be obtained in the direct space by applying a Fresnel free-space propagation:

$$
\begin{aligned}
\Psi_{z}(x, y)= & \Psi_{0}(x, y) \circledast \frac{1}{\mathrm{i} \lambda z} \exp \left(\mathrm{i} \pi \frac{x^{2}+y^{2}}{\lambda z}\right) \\
= & \frac{\varphi_{z}(x, y)}{\mathrm{i} \lambda z} \iint \Psi_{0}(\xi, \eta) \varphi_{z}(\xi, \eta) \\
& \times \exp \left(-2 \mathrm{i} \pi \frac{x \xi+y \eta}{\lambda z}\right) \mathrm{d} \xi \mathrm{d} \eta
\end{aligned}
$$

with $\varphi_{z}(x, y)=\exp \left(\mathrm{i} \pi \frac{x^{2}+y^{2}}{\lambda z}\right)$.

The symbol $\circledast$ stands for the convolution, and $\varphi_{z}(x, y)$ is the parabolic approximation of the spherical wave originating from a radiant point-source at a distance $z$. Equation (1) is the socalled Fourier-Fresnel transformation of the function $\Psi_{0}(x, y)$, which describes the Huygens-Fresnel principle. The number $N_{F}$ of Fresnel zones corresponds to the number of times this phase term increases in $\pi$, and in our case, $N_{F}=R^{2} / \lambda z$, with $R$ the radius of the occulter. For ASPIICS, $N_{F} \sim 6400$ at $\lambda=$ $550 \mathrm{~nm}$, while it would be on the order of 15 for an exoplanet experiment. The expanded form of Eq. (1) shows the diffracted wave as a scaled Fourier transformation of the wave $\Psi_{0}(x, y)$ that is affected by the quadratic phase term $\varphi_{z}(x, y)$. Since Eq. (1) is a convolution, $\Psi_{z}(x, y)$ can be written as follows:

$\Psi_{z}(x, y)=\mathcal{F}^{-1}\left[\mathcal{F}\left[\Psi_{0}\right] \times \mathcal{F}\left[\frac{\varphi_{z}}{\mathrm{i} \lambda z}\right]\right]$ 
where $\mathcal{F}[$.$] and \mathcal{F}^{-1}[$.$] denote the 2 \mathrm{D}$ direct and inverse Fourier transformations from the domain $(x, y)$ to frequency domain $(u, v)$. The Fresnel filter is given by

$\mathcal{F}\left[\frac{\varphi_{z}}{\mathrm{i} \lambda z}\right](u, v)=\exp \left(-\mathrm{i} \pi \lambda z\left(u^{2}+v^{2}\right)\right)$

It is worth emphasizing that the formulation in Eq. (2) is valid for any occulter shape.

We recall the case of a perfectly circular sharp-edged occulter of radius $R$. For an impinging plane wave of unit amplitude, coming from the on-axis point-source at infinity, Eq. (1) can be expressed as a radial function:

$$
\begin{aligned}
& \begin{aligned}
\Psi_{z}(r) & =\frac{\varphi_{z}(r)}{\mathrm{i} \lambda z} \int_{R}^{\infty} 2 \pi \rho \exp \left(\mathrm{i} \pi \frac{\rho^{2}}{\lambda z}\right) J_{0}\left(2 \pi \frac{r \rho}{\lambda z}\right) \mathrm{d} \rho \\
& =1-\frac{\varphi_{z}(r)}{\mathrm{i} \lambda z} \int_{0}^{R} 2 \pi \rho \exp \left(\mathrm{i} \pi \frac{\rho^{2}}{\lambda z}\right) J_{0}\left(2 \pi \frac{r \rho}{\lambda z}\right) \mathrm{d} \rho
\end{aligned} \\
& \text { with } \quad r=\sqrt{x^{2}+y^{2}} .
\end{aligned}
$$

$J_{0}($.$) is the Bessel function of the first kind. This Hankel trans-$ formation consists of the exact analytic expression of Fresnel diffraction in that particular case. At this stage, a radial apodization of the occulter disk can be accounted for with the formulation given in Eq. (5). We refer to the work of Aime (2013) for a more extensive study and for the alternative use of the Lommel series. In the following, we consider the sharp-edged occulting disk as a reference case in order to validate the other models we investigated.

\subsection{Theoretical approach of Vanderbei et al.}

Although stellar coronagraphy consists of a quite different problematic compared to solar coronagraphy, the diffraction that occurs follows the same Fresnel regime, and the equations used to describe the waves are similar. From the Fresnel theory, Vanderbei et al. (2007) investigated an analytical formulation to compute Fresnel diffraction for a petal-shaped occulter. Based on the procedure of Jacobi-Anger expansion (Vanderbei et al. 2003), $\Psi_{z}$ is written as the sum of Hankel transformations with high-orders Bessel functions.

We tried to apply this approach to our solar case, the toothed disk being a more elementary version of the petal-shaped disk. We define the serrated occulter by its number of teeth, denoted as $N_{t}$, its inner radius $R$, and the tooth height $\Delta$. Using the notations of our study, Eq. (9) of Vanderbei et al. (2007) becomes

$$
\begin{aligned}
\Psi_{z}(r, \theta)= & \Psi_{z}^{\mathrm{apod}}(r)-\frac{4}{\mathrm{i} \lambda z} \times \varphi_{z}(r) \times \sum_{j=1}^{\infty} \frac{(-1)^{j}}{j} \cos \left(j N_{t}(\theta-\pi / 2)\right) \\
& \times \int_{0}^{R+\Delta} \exp \left(\frac{\mathrm{i} \pi}{\lambda z} \rho^{2}\right) J_{j N_{t}}\left(\frac{2 \pi r \rho}{\lambda z}\right) \sin (j \pi f(\rho)) \rho \mathrm{d} \rho, \quad(5)
\end{aligned}
$$

where $\Psi_{z}^{\text {apod }}(r)$ is the Fresnel diffraction wave produced by the smoothed apodized occulter, the substitute of which is the serrated occulter. The related radial transmission function is $1-$ $f(r)$, as it appears in Eq. (5). This can be computed by a pseudoanalytical integration of Eq. (5), for instance. We recall that $\varphi_{z}(r)$ is given in Eq. (1). For a serrated occulter, the transmission is fully opaque in the central part, so that the factor $\sin (j \pi f(\rho))$ is zero when $\rho \leq R$. Thus, the integration boundaries in Eq. (5) can be reduced to $[R, R+\Delta]$.

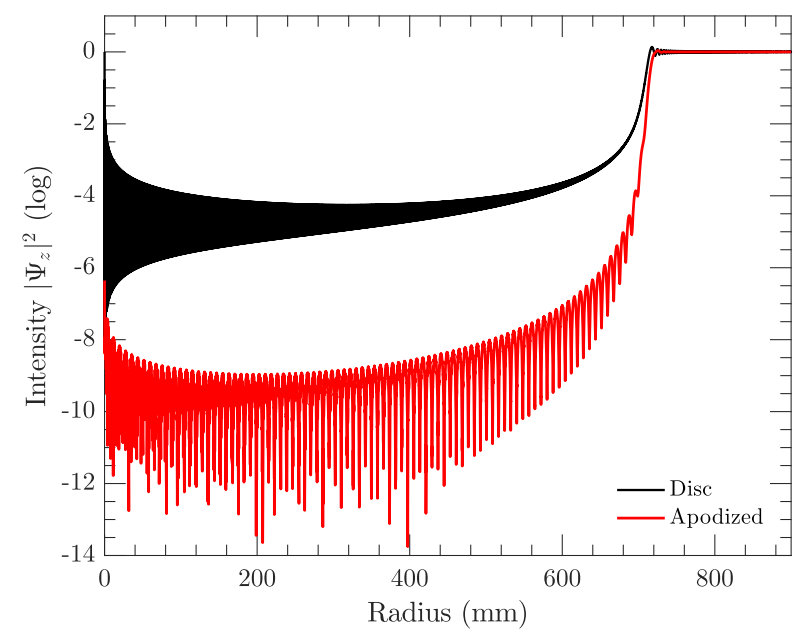

Fig. 1. Red: Fresnel diffraction intensity $\left|\Psi_{z}^{\text {apod }}(r)\right|^{2}$ of the apodized occulter. The transmission evolves linearly from 0 at $710 \mathrm{~mm}$ radius to 1 at $720 \mathrm{~mm}$. Black: sharp-edged disk as a reference.

The functions $J_{j N_{t}}$ become exponentially small near the center of the shadow for large $N_{t}$ when $j>0$. As the inner working angle is relatively small for exoplanet detection, the formulation in Eq. (5) is appropriate for computation, as only the first term $\Psi_{z}^{\text {apod }}$ dominates and the sum is neglected (Vanderbei et al. 2003). However, in our solar case, the field is much larger and the number of teeth usually on the order of several hundreds. We can no longer neglect the high-order Bessel functions, which renders the computation very complex and a delicate operation. Numerical implementation did not provide any substantial results because we were unable to make the sum converge. This approach was therefore discarded for our study.

To illustrate this discussion, Fig. 1 plots a cut of the diffraction intensity $\Psi_{z}^{\text {apod }}(r)$ of a circular apodized occulter. The transmission evolves linearly from 0 at $R=710 \mathrm{~mm}$ to 1 at $R+\Delta$, with $\Delta=10 \mathrm{~mm}$. The computation is based on the Hankel transformation as given in Eq. (5). The umbra level is much darker than the simple disk, by a gain of about six orders of magnitude.

\subsection{Numerical 2D FFT technique}

The Fresnel diffraction Eq. (2) can be implemented as a brute force numerical integration. We computed the 2D fast Fourier transformation (FFT) of an array of $K \times K$ sampling the external occulter, then multiplied this quantity with the Fresnel filter $\exp \left(-\mathrm{i} \pi \lambda z\left(u^{2}+v^{2}\right)\right)$, and finally returned to the real plane taking the inverse Fourier transformation. We used regular FFT algorithms, which made the spatial sampling $\sigma$ the same on the initial and final arrays.

With $K \times K$ points and a sampling $\sigma$, the occulter is padded in a square finite region $L \times L$, with $L=K \times \sigma$. We have an unusual padding here, since the occulter of transmission 0 is padded in a field of 1 . Now, we must address the sampling problem for Eq. (2).

A first point concerns the occulter itself. Our reasoning is based on the case of the sharp-edged disk. The Fresnel diffraction figure at a distance $z$ is the well-known Arago spot. At the center, its amplitude is equivalent to $J_{0}(2 \pi R r / \lambda z)$, as shown in Aime (2013). We now consider the reverse Fourier transformation of this function. Mathematically, it is a circle of radius $R / \lambda z$. In the Fourier space, the maximum spatial frequency is given by $1 / 2 \sigma$. This quantity must be larger than $R / \lambda z$, otherwise the 
circle overlaps and thereby aliasing effects occur. We derive thus a first condition:

$\sigma<\frac{\lambda z}{2 R}$.

With ASPIICS parameters, we find $\sigma<55.9 \mu \mathrm{m}$

A second consideration comes from the 1-or- 0 occulter, which provides thus a discontinuity in transmission. In the sense of a Fourier analysis, such an object is not band limited. The Shannon-Nyquist criterion is not applicable here. To mitigate this problem, we imposed that the spatial extent $L$ has to be much larger than the diameter of the occulter, in order to perform 1padding and avoid too strong aliasing effects when computing the Fourier transformation,

$K \times \sigma \gg 2 R$,

where the relation $\gg$ must be understood as larger than a factor 2 . We highlight that this condition is less critical when considering an apodized occulter because the sharpness of the edge is smoothed.

The third sampling problem concerns the Fresnel filter of the form $\exp \left(-\mathrm{i} \pi \lambda z u^{2}\right)$. It is a function showing extremely fast variation when $u$ increases, and therefore it is very difficult to sample. To avoid sampling problems, the approach of Mas et al. (1999) might be followed in their otherwise very interesting study of Fresnel diffraction, and a frequency cutoff might be assumed, which means setting the spectrum to zero after some given frequency. We tested applying a low-pass filter to the Fresnel spectrum. The diffraction pattern was smoothed, but the computed result was not satisfactory in the case of the simple disk: the Arago spot was degraded. We therefore chose not to follow their approach. The alternative would be to neglect the sampling condition, but then strong aliasing effects appear, which makes any subsequent calculation irrelevant because it propagates the wave inside the coronagraph. We report below the condition that must be imposed to perform a satisfactory sampling on the basis of the Fresnel filter.

After the FFT routine is applied, the sampling step in the Fourier plane is $1 /(K \times \sigma)$, and the highest frequencies in $u$ and $v$ are $u_{c}=v_{c}= \pm 1 /(2 \sigma)$. We assumed for convenience that a shift is applied, and that the zero of the FFT transformation is at the middle of the array, at the point $(K / 2+1, K / 2+1)$. We considered the $u$ direction of the axes for simplicity, and let $k$ be a point of the array in the region $u>0$. We recall that $k=0$ at $K / 2+1$ of the array. The corresponding frequency at point $k$ is $u_{k}=k /(K \sigma)=u_{c} k /(2 K)$. The value of the Fresnel filter at this point is thus

$\exp \left(-\mathrm{i} \pi \lambda z u_{k}^{2}\right)=\exp \left(-\mathrm{i} \pi \lambda z \frac{k^{2}}{(K \sigma)^{2}}\right)$.

Again, it is difficult to use Shannon's theorem directly for this problem, since the Fresnel filter is not band limited. However, a suitable criterion is that the phase difference of this quadratic filter does not exceed $\pi$ between two successive points. This gives the value of $k$ such that for $k+1$ we have an increase in phase no greater than $\pi$. We obtain

$\pi \lambda z \frac{(k+1)^{2}}{(K \sigma)^{2}}-\pi \lambda z \frac{k^{2}}{(K \sigma)^{2}} \sim \pi \lambda z \frac{2 k}{(K \sigma)^{2}} \leq \pi$.

This variation of $\pi$ is required to occur at the edge of the array, that is, for $k=K / 2$, and we derive the following relation:

$K=\frac{\lambda z}{\sigma^{2}} \Longleftrightarrow \sigma=\sqrt{\frac{\lambda z}{K}}$.
Table 1. Parameters for the 2D FFT technique. See text for details.

\begin{tabular}{lllll}
\hline \hline$K$ & $2^{16}$ & $2^{17}$ & & $2^{18}$ \\
\hline & 65536 & 131072 & 156000 & 262144 \\
$\sigma=\sqrt{\lambda z / K}$ & $34.8 \mu \mathrm{m}$ & $24.6 \mu \mathrm{m}$ & $22.6 \mu \mathrm{m}$ & $17.4 \mu \mathrm{m}$ \\
$L=K \times \sigma$ & $2.28 \mathrm{~m}$ & $3.22 \mathrm{~m}$ & $3.53 \mathrm{~m}$ & $4.56 \mathrm{~m}$ \\
$K \sigma / 2 R$ & 1.61 & 2.27 & 2.49 & 3.21 \\
\hline
\end{tabular}

It is interesting to note that when we inject Eq. (10) into Eq. (6), we find $L=K \times \sigma<2 R$, which simply means that the array must encompass the occulter. In practice, $K$ is very large, and we used the highest value that did not saturate the computer memory. In the present study, $K=156000$, and the sampling $\sigma$ is derived from Eq. (10). We verified that Eqs. (6) and (7) were satisfied and that the computation was accurate enough. Table 1 gives several possible sets of parameters.

Rougeot et al. (2017) propagated the diffracted wave further through the coronagraph and discussed another sampling condition. The reasoning was to have the same number of points padding the diameter of the telescope and padding the image of the external occulter formed by the objectives. In the hybrid case of ASPIICS, we recall that the Lyot mask (or internal occulter) is set at this particular image plane, instead of at the exact focal plane where the solar image is located, as for the classical Lyot coronagraph. If the propagation of the diffracted waves through the coronagraph is to be computed, this additional sampling condition of Rougeot et al. (2017; their Eq. (13)) needs be taken into account. We leave this for future work.

As a reference test case, we considered the circular sharpedged occulter, in order to validate the 2D FFT technique. Figure 2 plots a mere central cut of the 2D diffraction pattern, which we compare to the reference (Eq. (5)) as given in Fig. 2 of Rougeot et al. (2017). We used an array of size $K=156000$ and a spatial sampling of $\sigma=22.6 \mu \mathrm{m}$. Panel a shows the full pattern extended in logarithmic scale; panel b shows a zoom in the central spot in linear scale, whose peak intensity is 1 for $r=0$, as expected; panel c shows the transition zone between shadow and light. As expected, the numerical FFT results present small noisy fluctuations. The FFT results are also very similar to the reference, despite the low sampling of $22.6 \mu \mathrm{m}$; this is clearly visible in panel b. As our main objective is to compute the penumbra profile by integration over the solar disk, which has a smooth pattern, this is not a critical concern. Finally, we may investigate the effect of deviating from the sampling condition (10). This is reported in Appendix A.

To conclude, the brute force 2D FFT technique seems adequate to compute Fresnel diffraction, provided that the size of the array $K$ is large enough and that the sampling condition Eq. (10) is fulfilled. We tested this method for serrated occulters and describe the results below.

\subsection{Maggi-Rubinowicz representation}

An alternative approach to computing the diffracted wave behind a screen or an aperture is given by the Maggi-Rubinowicz representation. Instead of the 2D integral of the Fresnel formulation, this method relies on a single boundary integral along the edge of the diffracting object. It must be noted that this modeling is only suitable for the 1 -or- 0 mask, so no apodization can be investigated.

The analytical derivation of this approach is well described in Born \& Wolf (2006) based on the Kirchhoff integral theorem. 

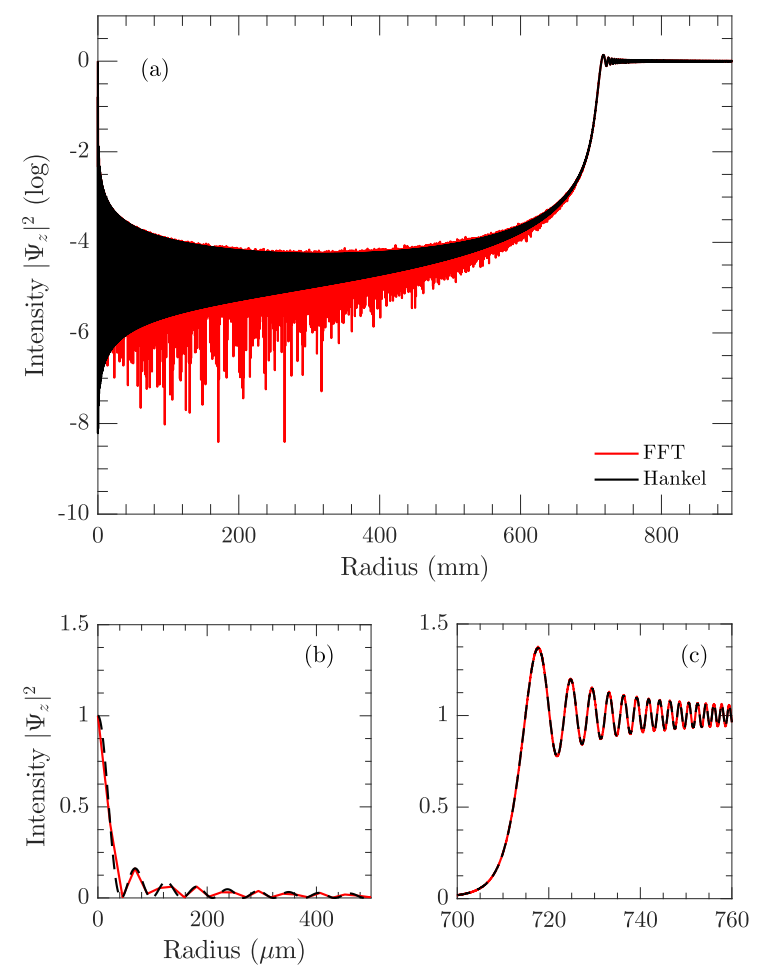

Fig. 2. Red: Fresnel diffraction intensity $\left|\Psi_{z}(r)\right|^{2}$ from the $2 \mathrm{D}$ FFT computation Eq. (2). Black shows the Hankel computation Eq. (5) as a reference. The occulter is a sharp-edged disk of radius $710 \mathrm{~mm}$ set at $z=144.348 \mathrm{~m}$. An array of $156000 \times 156000$ and a sampling $\sigma=22.6 \mu \mathrm{m}$ have been used. Panel $a$ : full range, in logarithmic scale. Panel b: zoom in the Arago bright spot in the central region [0,500 $\mu \mathrm{m}]$, in linear scale. Panel $c$ : transition region between shadow and light around $710 \mathrm{~mm}$, in linear scale.

We consider an incoming wave $\Psi_{0}$ that is diffracted by an aperture $\mathcal{A}$ lying on an infinite opaque screen. We denote by $\Psi_{z}^{\mathrm{Ap}}(x, y)$ the complex amplitude of the wave at a point $(x, y, z)$ downstream of the aperture. It is split between two terms

$\Psi_{z}^{\mathrm{Ap}}(x, y)=\Psi_{z}^{(g)}(x, y)+\Psi_{z}^{(d)}(x, y)$,

where $\Psi_{z}^{(g)}(x, y)$ consists of the wave as dictated by the geometrical optics, that is, $\Psi_{0}$ in the extent of the opening and 0 otherwise, and $\Psi_{z}^{(d)}(x, y)$ is the diffraction disturbance. This last term is written as a curvilinear integral along the boundary $\partial \mathcal{A}$ of the aperture.

In the framework of stellar coronagraphy, Cady (2012) formulated this boundary diffraction wave integral by introducing a potential vector and applying the Stokes theorem. As an occulter is the complementary of an opening in an opaque screen, the Babinet theorem is applicable. The complex amplitude $\Psi_{z}^{\text {Occ }}(x, y)$ of the diffracted wave behind the occulter, at the same point $(x, y, z)$, is thus linked to $\Psi_{z}^{A p}(x, y)$ following

$\Psi_{z}^{\mathrm{Ap}}(x, y)+\Psi_{z}^{\mathrm{Occ}}(x, y)=\Psi_{0}$.

Equations (11) and (12) give the expression of the diffraction by the occulter:

$\Psi_{z}(x, y)=-\Psi_{z}^{(d)}(x, y)$ in the geometrical shadow,

$\Psi_{z}(x, y)=\Psi_{0}-\Psi_{z}^{(d)}(x, y)$ otherwise.

We verified that the geometrical derivation of Born \& Wolf (2006) and the vectorial representation presented in Cady (2012) are consistent. Considering an impinging wave from the on-axis point-source at infinity, we have $\Psi_{0}=1$. The final expression of the disturbance is given by

$\Psi_{z}^{(d)}(x, y)=\frac{1}{4 \pi} \int_{\partial \mathcal{A}} \frac{\exp \left(\frac{2 \mathrm{i} \pi}{\lambda}(s-z)\right)}{s-z} \times \frac{-(\xi-x) l_{\eta}+(\eta-y) l_{\xi}}{s} \mathrm{~d} l$

where $\boldsymbol{l}=\left(\boldsymbol{l}_{\xi}, \boldsymbol{l}_{\eta}, \mathbf{0}\right)$ is the unit vector in the direction tangent to the edge at any point $P=(\xi, \eta, 0)$ of $\partial \mathcal{A}, s$ is the distance between $P$ and the observation point $(x, y, z)$, and $\mathrm{d} l$ is the differential curvilinear element of the edge. This mathematical expression can be expanded given a specific occulter edge $\partial \mathcal{A}$ and related vector $\boldsymbol{l}$. Because occulters usually have a certain cylindrical periodicity, it is convenient to write Eq. (14) using polar coordinates $(\rho, \phi)$ in the integrand instead of $(\xi, \eta)$, and perform the integration on $\phi \in[0 ; 2 \pi]$, either by discretization or analytical integration. In contrast to the FFT technique, this approach does not require a discussion of the sampling. No aliasing effects are present, which is intrinsically linked to the way the FFT works.

The detailed expression of the sharp-edged disk is given by Eq. (B.2). The computation was performed by directly sampling the edge of the disk, that is, the angle $\phi$, using 100000 points. Figure 3 plots the diffraction intensity $\left|\Psi_{z}(r)\right|^{2}$, which we compare to the same reference Eq. (5) that is obtained using NIntegrate of Mathematica. Panels a-c show the same as Fig. 2. The diffraction pattern is very smooth and agrees perfect well with the reference. However, a divergence around the transition between shadow and light at $r \sim 710 \mathrm{~mm}$ is observed because $s(\phi=0)$ tends to $z$ when $r \sim R$; see Eq. (B.2). In addition to this feature, we considered the MaggiRubinowicz representation to compute diffraction for serrated occulters.

An interesting result of the Rubinowicz representation for the simple occulting disk is the value reached by the intensity at the center, the so-called Arago bright spot. From Eq. (B.2), we obtain analytically

$\left|\Psi_{z}(0)\right|^{2}=\frac{R^{4}}{4 R^{2}\left(z-z\left(z-\sqrt{z^{2}+R^{2}}\right)\right)^{2}}$.

With ASPIICS parameters, we find a value of 0.99995, which differs from the well-known unitary spot, as obtained using Eq. (5), for instance. Very surprising at first, this discrepancy comes from the parabolic approximation used in Fresnel diffraction equations, which is not assumed by the Rubinowicz representation. When we expand the square root in Eq. (15) in terms of $R^{2} / z^{2}$, we obtain the expected value of 1 .

\section{Diffraction pattern of serrated occulters}

We now consider thin serrated occulters in the geometrical configuration of ASPIICS coronagraph. We recall that the disk is defined by its number of teeth, denoted as $N_{t}$, its inner radius $R$, and the size of the teeth $\Delta$ of a few centimeters. The computational results were obtained with a machine with two 14-core Intel Xeon processors and 512 GB of RAM.

\subsection{Numerical validation}

We first validated the numerical computation for the serrated occulters. In the Rubinowicz formulation Eq. (14), the curvilin- 

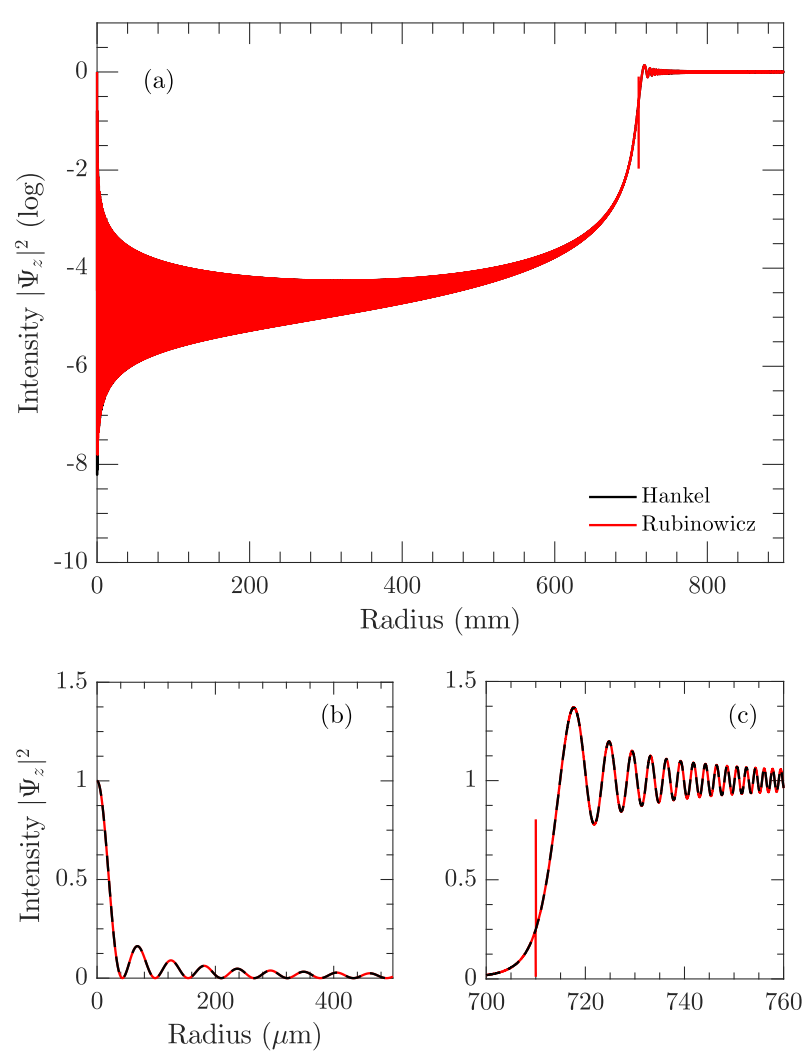

Fig. 3. Red: Diffraction intensity $\left|\Psi_{z}(r)\right|^{2}$ using the Rubinowicz representation. Black shows the Hankel computation Eq. (5) as a reference. The occulter is a sharp-edged disk of radius $710 \mathrm{~mm}$ set at $z=$ $144.348 \mathrm{~m}$. Panel $a$ : full range, in logarithmic scale. Panel $b$ : zoom in the Arago bright spot in the central region $[0,500 \mu \mathrm{m}]$, in linear scale. Panel c: transition region between shadow and light around $710 \mathrm{~mm}$, in linear scale.

ear integral along the occulter can be performed either analytically or by discretization. The related expanded expression is given in Appendix B. Near the central point, we have observed that the integrand oscillates very quickly with the integration variable $\phi$. This numerical consideration does not encourage the use of analytical integration technique, and we finally chose to compute the integral (Eq. (14)) by sampling the edge. We did not find any simple condition to correctly sample the occulter edge in the literature. Therefore, our approach is somewhat empirical.

We first imposed the condition that there is a sampling point at every peak and trough of the teeth. We denote by $\sigma_{R}$ the spatial sampling along the edge of the occulter, that is, the distance between two successive points for the integration. This is different from the angular sampling $\mathrm{d} \phi$. By reducing $\sigma_{R}$, which means increasing the number of points along the edge, we intuitively know that the resulting diffraction curve will be more accurate, but will also require more computational time. We aim to find a condition that gives a reasonable value for $\sigma_{R}$. We recall that we used 100000 points for the disk, which corresponds to $\sigma_{R}=44.6 \mu \mathrm{m}$, and the result was very satisfactory. We focused on the particular 1024-tooth occulter, which is the most stringent with respect to the number of teeth. We verified that reducing $\sigma_{R}$ causes the computed diffraction curves to converge. The average relative difference between the computations using $\sigma_{R}=10 \mu \mathrm{m}, 5 \mu \mathrm{m}$, and $2 \mu \mathrm{m}$, with respect to the computation using $\sigma_{R}=1 \mu \mathrm{m}$, are $2.4 \%, 0.55 \%$, and $0.07 \%$, respectively. Therefore we set as empirical criteria $\sigma_{R}=2 \mu \mathrm{m}$, which seems

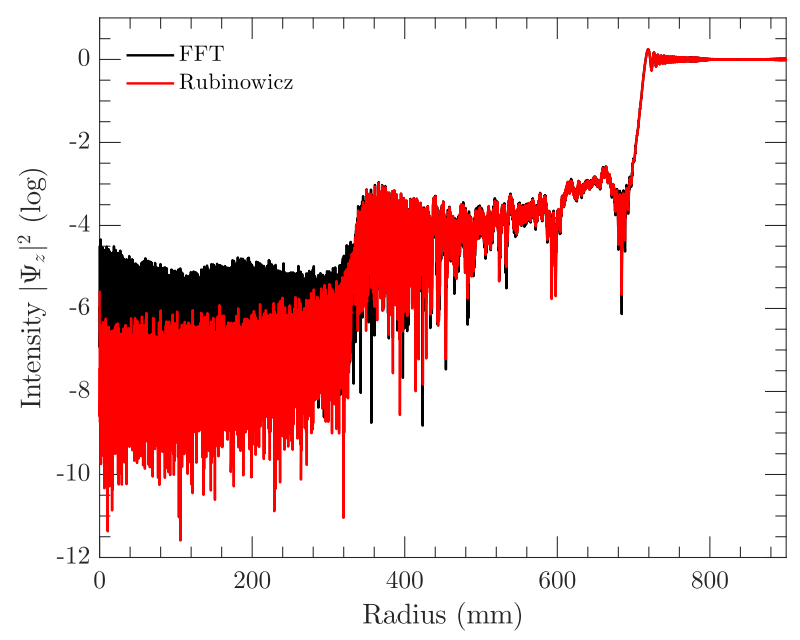

Fig. 4. Horizontal cuts of the diffraction intensity $\left|\Psi_{z}(x, y)\right|^{2}$ for a serrated occulter of 64 teeth of $20 \mathrm{~mm}$, in logarithmic scale. Black shows the computation using the 2D FFT technique. Red depicts the computation using the Rubinowicz method. See text for comments.

reasonable. This value is much lower than the value we used for the disk.

We now compare the 2D FFT technique and the Rubinowicz method for serrated occulters. The FFT routine uses an array $K \times K=156000 \times 156000$ and a sampling $\sigma=22.6 \mu \mathrm{m}$. We illustrate the comparison in this paper by using a serrated occulter of 64 teeth of $20 \mathrm{~mm}$. Figure 4 plots the cuts of the intensity $\left|\Psi_{z}(x, y)\right|^{2}$ along the $x$-axis, obtained by both computations, in logarithmic scale. The two curves strongly differ in the central region of the diffraction pattern, but they coincide in an outer region where the intensity is brighter. On the one hand, as we previously identified, the accuracy of the FFT routine strongly depends on the size of the array because the computational memory is limited. Considering that the FFT analysis can be made only for a limited field, we may explain the observed discrepancy by an overlapping of the computed 2D images. On the other hand, the Rubinowicz method has proven to be rather efficient and accurate. For our present study, we legitimately decided to discard the 2D FFT technique, mostly because of our computational resources were limited.

In the following, the diffraction was computed using the Rubinowicz method, with a spatial sampling $\sigma_{\mathrm{R}}=2 \mu \mathrm{m}$ along the occulter edge. However, the use of the 2D FFT technique remains valid in the region relatively close to the transition from shadow to light.

\subsection{Analysis of the diffraction pattern}

Figure 5 shows the shape of a few occulters, and the modulus and phase of the related complex amplitude $\Psi_{z}(x, y)$ near the edge of the Fresnel pattern of the occulter. The representation is limited to a rectangular zone of $80 \mathrm{~mm} \times 60 \mathrm{~mm}$, and the central position is at $720 \mathrm{~mm}$, which is already outside the projected solar limb of about $671 \mathrm{~mm}$ radius. Panel a shows the sharp-edged disk, as a reference; panels b and c show serrated occulters defined by $N_{t}=512, \Delta=10 \mathrm{~mm}$ and $N_{t}=1024$, $\Delta=20 \mathrm{~mm}$, respectively. We here study the transition zone near the edge and therefore computed the 2D diffraction patterns using the 2D FFT technique, which was proven to be valid in this region.

Figure 6 plots several cuts of the intensity $\left|\Psi_{z}(x, y)\right|^{2}$ along the $x$-axis in logarithmic scale for a 64-, 512-, and 1024-tooth 
R. Rougeot and C. Aime: Theoretical performance of serrated external occulters for solar coronagraphy
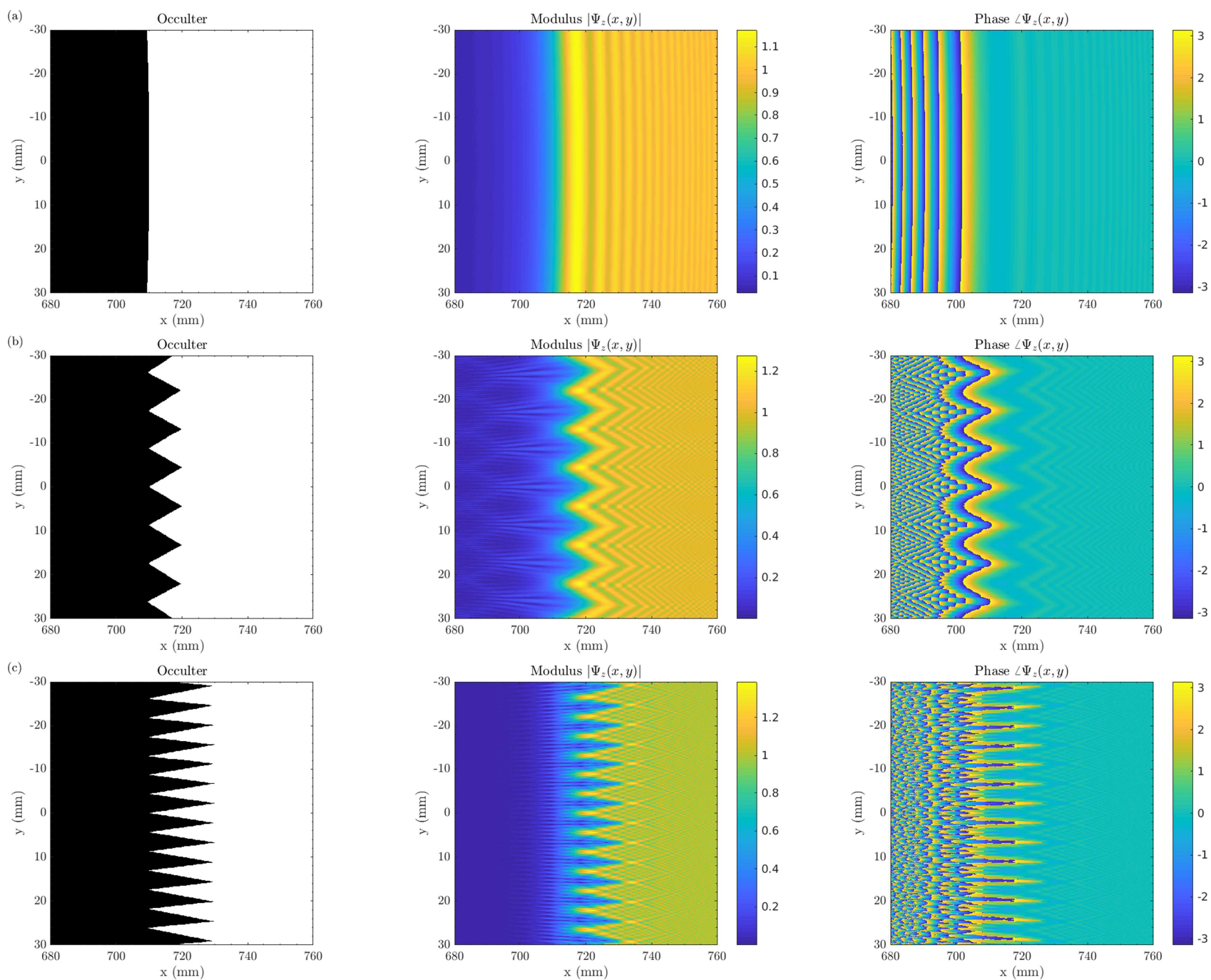

Fig. 5. 2D representation of the occulter, the modulus $\left|\Psi_{z}(x, y)\right|$, and the phase $\angle \Psi_{z}(x, y)$ of the diffraction. The transition region between light and shadow is a rectangular zone of $80 \mathrm{~mm} \times 60 \mathrm{~mm}$ with a central position at $720 \mathrm{~mm}$. From top to bottom: Panel a: circular sharp-edged occulter. Panel $b$ : serrated occulter $N_{t}=512$ and $\Delta=10 \mathrm{~mm}$. Panel $c$ : serrated occulter $N_{t}=1024$ and $\Delta=20 \mathrm{~mm}$.

disk; see the legends of the plots. The sharp-edged disk is plotted behind as a reference. We also show the intensity of the corresponding apodized occulter, whose transmission extends linearly from 0 at $R$ to 1 at $R+\Delta$. Numerically, the Rubinowicz computation contains only very few divergence features, as was observed for the disk. Here, only one or two sampling points are invalid (either infinity or "not a number"), and they were removed. This model remains very satisfactory.

Several comments and observations can be made based on these results. First, we observe that the diffraction patterns produced by serrated occulters present a two-level dark region, the inner being the darker. The spatial extent of this inner dark central zone strongly varies with the number and the size of the teeth. For instance, it is about $180 \mathrm{~mm}$ long for the 64-tooth disk with $10 \mathrm{~mm}$ teeth, but up to $700 \mathrm{~mm}$ for the 1024-tooth disk with $30 \mathrm{~mm}$ teeth. In general, the larger $N_{t}$ and $\Delta$, the longer the dark central zone.

Starting from the idea of Purcell \& Koomen (1962) that the edges of the teeth reject the light outside the central part of the dark shadow of the occulter, Boivin (1978) reported the expected radius of this dark inner zone that is produced by the serrated occulter using geometrical considerations. According to his Eq. (1)a, this radius that we denote $B_{N_{t}, \Delta}$ can be written as

$B_{N_{t}, \Delta}=R \cos \left(\Lambda+\hat{N}_{1 / 2}\right)$,

where $\Lambda$ is the half-apex angle of the teeth, and $\hat{N}_{1 / 2}=\pi / N_{t}$ is half the angular separation of two successive teeth, that is, the angle under which the half-tooth is seen from the center of the occulter. The angle $\Lambda$ is derived from the geometry of the system, as given in Eq. (1b) of Boivin (1978),

$\Lambda=\arctan \left(\frac{R \sin \hat{N}_{1 / 2}}{R\left(1-\cos \hat{N}_{1 / 2}\right)+\Delta}\right)$.

Figure 7 plots the Boivin radius function of the number of teeth for different tooth heights. As we see in Fig. 6, the Boivin-predicted radii plotted as vertical lines are in very good concordance with the computed diffraction patterns and with the related observation we made.

The diffraction intensity level can reach about $10^{-8}$, with a high number of teeth. This is much better than the sharp-edged disk; the gain is from two to four orders of magnitude in the 

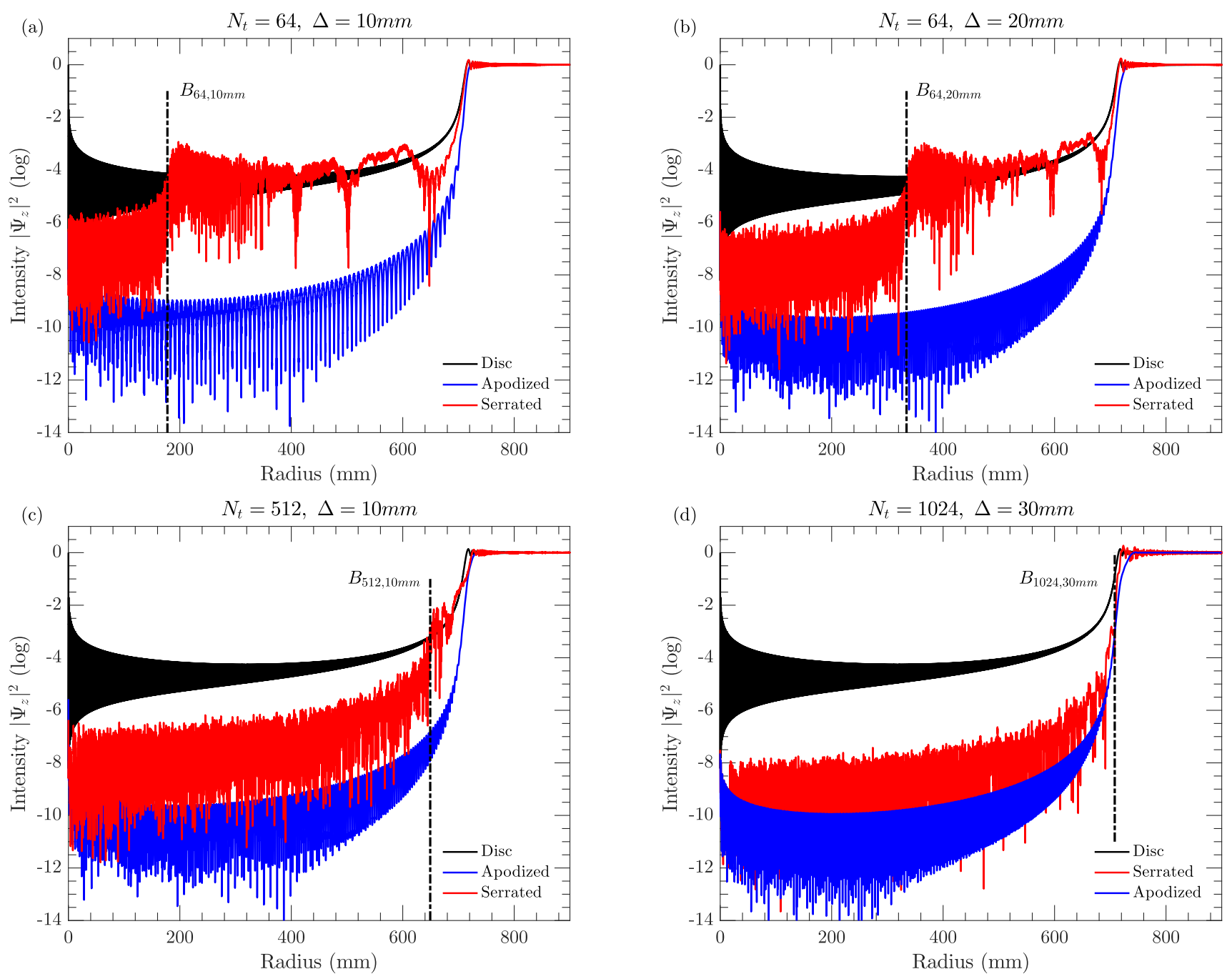

Fig. 6. Red: cuts of the diffraction intensity $\left|\Psi_{z}(x, y)\right|^{2}$ for several serrated occulters in logarithmic scale. The vertical lines shows corresponding Boivin radii $B_{N_{T}, \Delta}$. Black: sharp-edged disk. Blue: apodized disk. Panel a: $N_{t}=64, \Delta=10 \mathrm{~mm}$. Panel b: $N_{t}=64, \Delta=20 \mathrm{~mm}$. Panel $\mathrm{c}: N_{t}=512$, $\Delta=10 \mathrm{~mm}$. Panel $d: N_{t}=1024, \Delta=30 \mathrm{~mm}$.

central region. However, the apodized occulter remains the best in terms of rejection performance, but many long teeth allow one to come close to this level; see panel d of Fig. 6.

Finally, we investigated the effect of rotating the serrated occulter about its axis by computing the diffraction either along a line with a tooth trough or a line with a peak. This is illustrated in Fig. 8, where we used an occulter of 64 teeth. The diffraction curves are very similar inside Boivin's central region, and they differ outside of it. The transition between shadow and light is shifted by a distance $\Delta$, as expected.

As an additional analysis of the diffraction intensity, Appendix $\mathrm{C}$ treats the small variations with wavelength at the center of the umbra.

\section{Rejection performance of serrated occulters}

\subsection{Umbra profile}

Aime (2013) computed the penumbra profile $I_{z}(x, y)$ on the plane at $z$. This consists of the convolving the diffraction intensity $\left|\Psi_{z}(x, y)\right|^{2}$ with the solar disk. Here, the assumption is that

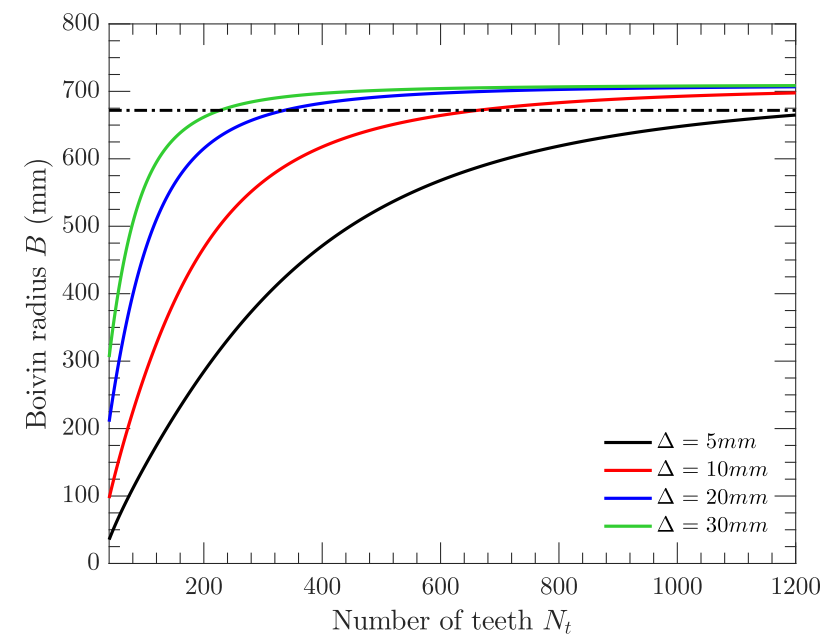

Fig. 7. Boivin-predicted radius $B_{N_{t}, \Delta}$ as a function of the number $N_{t}$ for different tooth heights $\Delta$. The horizontal black dotted line at $671 \mathrm{~mm}$ corresponds to the projected stenope radius of the Sun for the geometry of ASPIICS. 

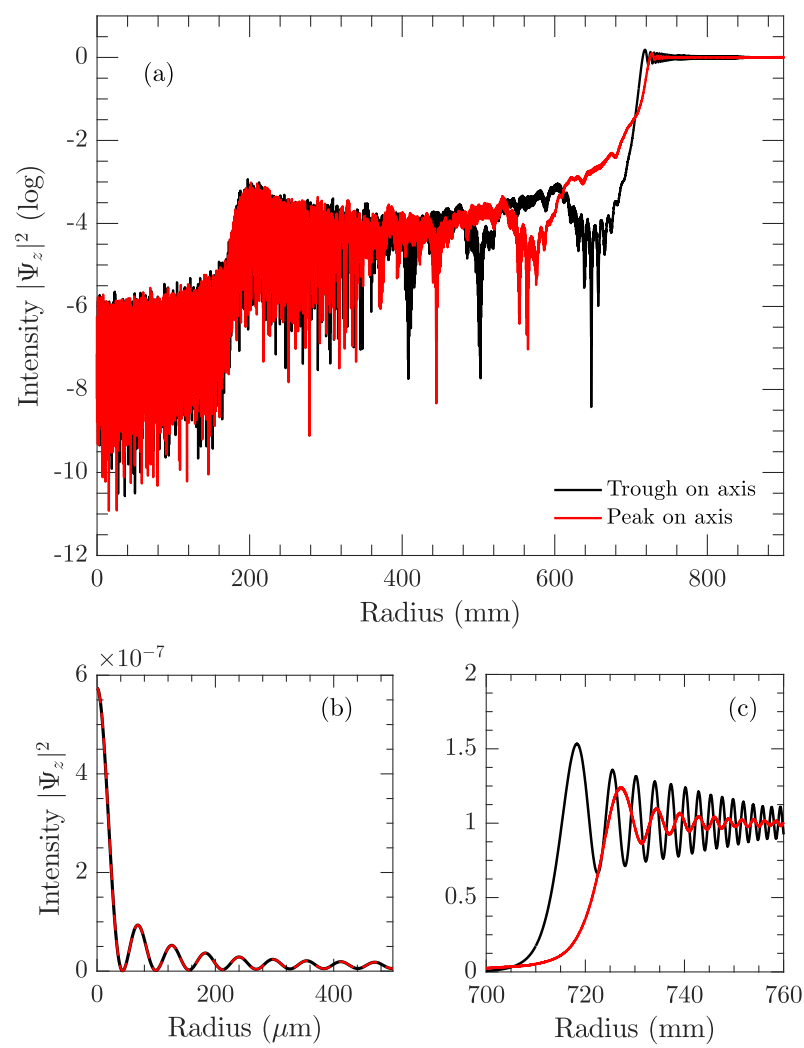

Fig. 8. Two cuts of the diffraction intensity $\left|\Psi_{z}(x, y)\right|^{2}$, one along a peak of a tooth, one along a trough, for a serrated occulter with 64 teeth of $10 \mathrm{~mm}$. Black shows the trough of a tooth on the axis. Red plots the peak of a tooth on the axis. Panel $a$ : full range, in logarithmic scale. Panel $b$ zoom in the central region $[0,500 \mu \mathrm{m}]$, in linear scale. Panel $c$ : transition region between shadow and light around $710 \mathrm{~mm}$, in linear scale.

the shape of the projected geometrical shadow of the occulter remains the same for every point-source describing the solar disk. In other words, we assume that $\cos 2 R_{\odot}$ is equal to 1 , the actual value being 0.9997 ,

$$
\begin{aligned}
I_{z}(x, y) & =\left|\Psi_{z}(x, y)\right|^{2} \circledast \mathcal{B}_{z}(x, y) \\
& =\iint\left|\Psi_{z}(\xi, \eta)\right|^{2} \times \mathcal{B}_{z}(x-\xi, y-\eta) \mathrm{d} \xi \mathrm{d} \eta,
\end{aligned}
$$

where $\mathcal{B}_{z}(x, y)$ denotes the $2 \mathrm{D}$ projected stenope image of the Sun, including the center-to-limb darkening. This function is radial and has a limited extent given by $r_{\odot}=z \times \tan R_{\odot}$, where $R_{\odot}$ is the angular radius of the Sun. In the case of ASPIICS, $r_{\odot}=671 \mathrm{~mm}$. We used the same center-to-limb variation of the Sun as Rougeot et al. (2017), which was derived from Hamme (1993).

We chose to compute only a radial penumbra profile along the $x$-axis, even if there is no actual axisymmetry for the serrated occulter. Equation (18) becomes

$$
I_{z}(r)=\int_{\sqrt{\xi^{2}+\eta^{2}} \leq r_{\odot}}\left|\Psi_{z}(r+\xi, \eta)\right|^{2} \times \mathcal{B}_{z}(\xi, \eta) \mathrm{d} \xi \mathrm{d} \eta .
$$

First of all, we can predict the theoretical rejection performance of serrated external occulters based on the discussion in Sect. 3. If the Boivin radius is large enough with respect to the projected solar radius, that is, $B_{N_{t}, \Delta}>r_{\odot}$, the convolution in Eq. (19) will integrate the solar image on the dark region

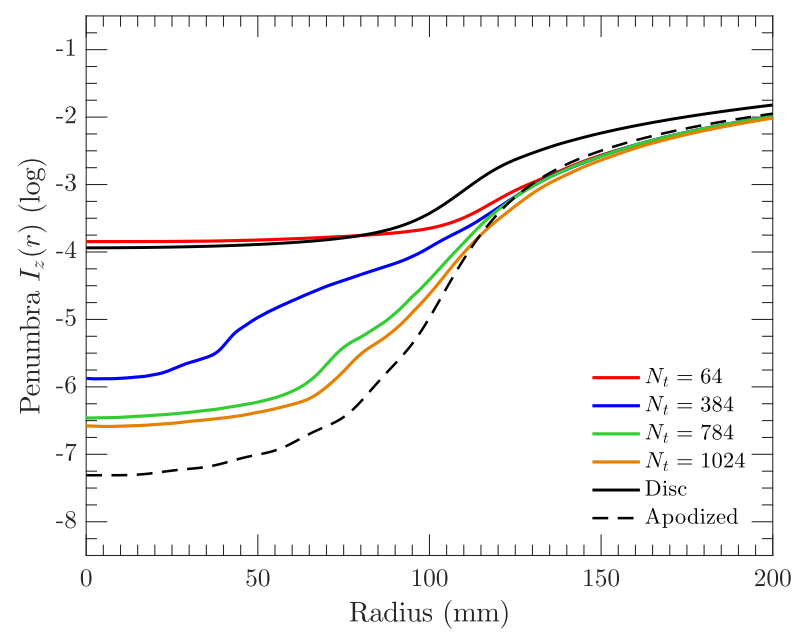

Fig. 9. Penumbra profile $I_{z}(r)$ for several serrated occulters with $20 \mathrm{~mm}$ teeth in logarithmic scale in the range $r \leq 200 \mathrm{~mm}$. Red shows $N_{t}=64$. Blue shows $N_{t}=384$. Green represents $N_{t}=784$. Yellow plots $N_{t}=1024$. Black shows the sharp-edged disk. The black dashed line represents the apodized disk.

of the two-level diffraction intensity. On the other hand, if the Boivin radius is rather small, the solar image overlaps the bright area. The resulting umbra intensity at the center will thus not be low - in a relative sense. In Fig. $7, r_{\odot}$ is plotted as a horizontal line to facilitate comparison. This deep relation between the Boivin radius and the theoretical umbra may give a first indication about the ideal serrated external occulter. We must now verify this prediction.

We computed the penumbra $I_{z}(r)$ for many serrated occulters up to $200 \mathrm{~mm}$ because the pupil is only some few centimeters in diameter, and we are not interested in the far penumbra. We preferred not to compute the full 2D diffraction patterns $\left|\Psi_{z}(x, y)\right|^{2}$, and chose to reconstruct it. To do so, we performed a linear interpolation using three different computations of the diffraction intensity on the $x$-axis: a tooth peak on the axis, a tooth trough on the axis, and the intermediate curve. This interpolation method was validated against the sharp-edged disk. We retrieved the umbra level of $10^{-3.94}$ and the same penumbra profile as computed and described in Rougeot et al. (2017).

First, we analyze the effect of the number of teeth, given a fixed tooth height. Figure 9 plots the penumbra for 64-, 384-, 784- and 1024-tooth disks with $\Delta=20 \mathrm{~mm}$. The sharp-edged and apodized disks are also plotted as a reference. As expected, the more teeth, the deeper the umbra. Surprisingly, the 64-tooth disk is slightly worse than the disk. In this particular case, the corresponding Boivin radius is small, and the diffraction intensity is brighter than that from the disk; see panel b of Fig. 6 . The improvement from 784 to 1024 teeth is small. In this latter case, the gain is about 2.5 orders of magnitude with respect to the sharp-edged disk, whereas the apodized disk remains the best. In the same way, we investigated the effect of the tooth size. Figure 10 plots the penumbra for a 484-tooth disk with 10 , 20, 30 and $40 \mathrm{~mm}$ teeth. Again as expected, the umbra deepens with increasing tooth size. A level of $10^{-7}$ is achieved with $\Delta=40 \mathrm{~mm}$, almost similar to the performance of the apodized occulter.

\subsection{Analysis and discussion}

In order to quantify the gain in rejection performance, we took as a criterion the total power of diffracted sunlight that would 


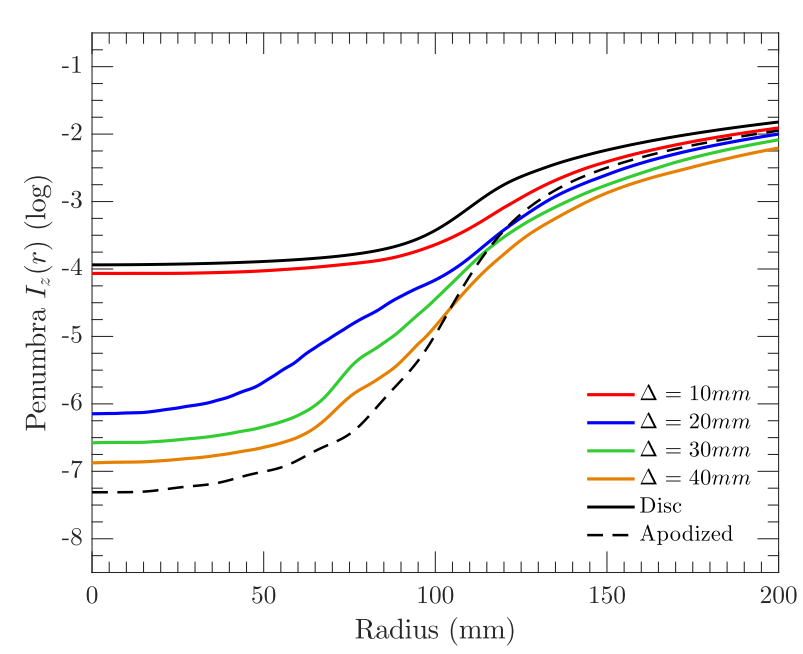

Fig. 10. Penumbra profile $I_{z}(r)$ for several serrated occulters $N_{t}=$ 464 teeth in logarithmic scale in the range $r \leq 200 \mathrm{~mm}$. Red shows $\Delta=10 \mathrm{~mm}$. Blue represents $\Delta=20 \mathrm{~mm}$. Green plots $\Delta=30 \mathrm{~mm}$. Yellow depicts $\Delta=40 \mathrm{~mm}$. Black shows the sharp-edged disk. The black dashed line shows the apodized disk.

enter the coronagraph. We computed the integrated umbra illumination $P_{z}$ over the entrance aperture of radius $R_{p}$, which is $25 \mathrm{~mm}$ for ASPIICS, which we normalized by the case of the sharp-edged disk. This criterion is defined as

$P_{z}\left(R_{p}\right)=\frac{\int_{0}^{R_{p}} I_{z}(\rho) \rho \mathrm{d} \rho}{\int_{0}^{R_{p}} I_{z}^{\operatorname{disc}}(\rho) \rho \mathrm{d} \rho}$.

Figure 11 shows the color map of the power $P_{z}\left(R_{p}\right)$ as a function of the number and the tooth size in logarithmic scale. The white line in the plot gives the pairs $\left(N_{t}, \Delta\right)$ such that $B_{N_{t}, \Delta}>r_{\odot}$ above. For sake of comparison, the apodized disk gives a value $P_{z}^{\text {apod }}\left(R_{p}\right)=10^{-3.35}$.

Our prediction is well verified: serrated occulters with a Boivin radius larger than the projected solar radius perform better than the disk. Few teeth, between 200 and 400, would require long teeth to compensate for this in order to gain in rejection. Even more, occulters with $N_{t}<200$ are worse than the disk. Conversely, occulters with 1000 teeth provide a substantial gain of two orders of magnitude, which is a factor 100 . This satisfying result proves the actual advantage of shaped occulters, which must be preferred to simple disks.

As a final result, we considered a serrated occulter made of $N_{t}=1080$ teeth, which consists of three teeth per angle degree. Such a tooth has base length of $4.13 \mathrm{~mm}$, which seems reasonable. The height was fixed to $\Delta=20 \mathrm{~mm}$ to avoid hiding too much of the inner solar corona. At the center, the umbra reaches a level $I_{z}(0)=10^{-6.61}$, and the rejection gain is $P_{z}\left(R_{p}\right)=10^{-2.65}$. The comparison with the apodized disk shows that the rejection from this latter is only better by a factor 5 . Serrated occulters can therefore match this ideal performance closely.

However, we recall that our study remains theoretical and numerical. The serrated occulters we have considered here are perfect, in the sense that all teeth are perfectly identical. The actual results would be subject to tolerancing and manufacture defects. As discussed in Fort et al. (1978), the edge must be super polished, and the measured performance can degrade very quickly. The rejection performance we have shown here must be viewed as an upper boundary of what can be reached in reality.

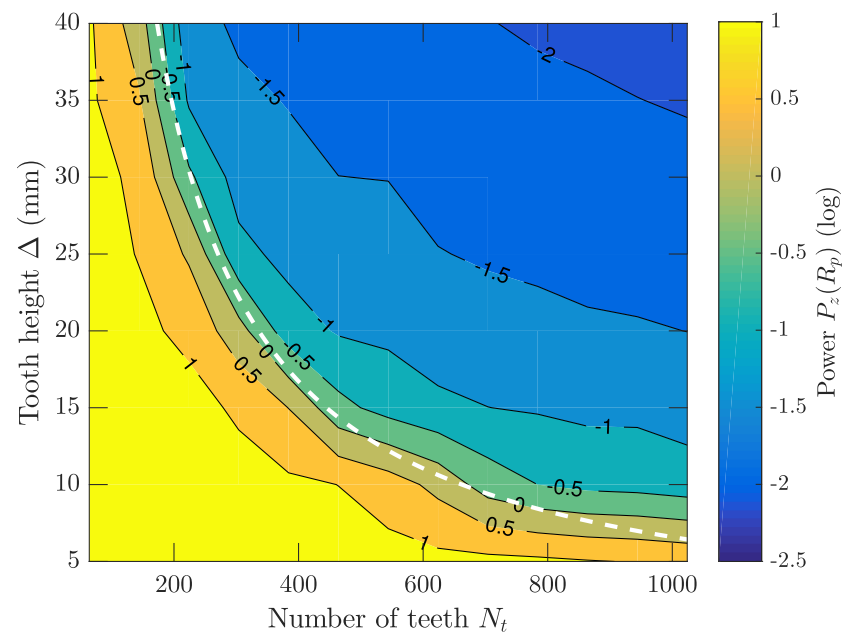

Fig. 11. Integrated penumbra illumination $P_{z}\left(R_{\text {pup }}\right)$ over the entrance aperture, function of the number of teeth $N_{t}$, and the teeth height $\Delta$ in logarithmic scale. The white line plots the pairs $\left(N_{t}, \Delta\right)$ such that $B_{N_{t}, \Delta}=r_{\odot}$.

\section{Conclusion}

We computed the diffraction patterns from the on-axis pointsource at infinity for various serrated occulters made of very many teeth of a few centimeters each. We showed that serrated occulters tend to create a two-level intensity, with the inner being the darker. Our results agree perfectly well with the prediction of Boivin (1978). The diffraction in this dark central region is lower by far than the diffraction produced by the sharp-edged disk.

We showed that the umbra deepness of serrated external occulters is closely related to the Boivin radius, as predicted. Increasing the number and height of the teeth substantially improves the rejection performance. With 1000 teeth, a serrated occulter can reach a gain in rejection of a factor 100 with respect to the disk, and it closely matches the performance of the apodized disk.

Nevertheless, we underline that our study remains theoretical and numerical. A first question concerns the actual feasibility of manufacturing such serrated occulters with very many teeth. Second, the actual performance is driven by the quality of the overall system. Any defects from tolerancing of the edge will degrade it. However, these defects would be purely mechanical, and it is probably easier to achieve a very good quality for the occulter itself, in comparison to what is required for the optics in the Lyot coronagraph. A comparison with an experimental approach would be of interest, as was reported by Landini et al. (2016).

Finally, we are interested in the complete end-to-end performance of the instrument that results from the combination of the external occulter and the hybrid Lyot coronagraph. The diffracted wavefront made by serrated occulters must be propagated through the coronagraph, in order to assess the residual level of sunlight on the final focal plane. Moreover, increasing the tooth size introduces vignetting, which may degrade the inner field of view. This would require a delicate image-reconstruction processing for observations of the corona very close to the solar limb. This study is left for future work.

In addition to the strict consideration of serrated occulters, we investigated diffraction modeling in the context of solar coronagraphy, and studied several numerical methods. The brute force FFT technique has the strong advantage of directly computing the $2 \mathrm{D}$ diffraction patterns, but it may unfortunately be 
R. Rougeot and C. Aime: Theoretical performance of serrated external occulters for solar coronagraphy

limited by available computational resources. As an alternative, the Maggi-Rubinowicz representation has proven to be very powerful for a 1 -or- 0 occulter, allowing us to integrate only along the edge. These diffraction models will enable us to investigate other types of occulters and to consider tolerancing and defects of the edge, for instance, in both solar and stellar coronagraphy.

Acknowledgements. We would like to thank R. Flamary and D. Mary for their help in this study. We are grateful to the anonymous referee for their time and suggestions to improve our paper. We also thank the European Space Agency and Thales Alenia Space for supporting this activity.

\section{References}

Aime, C. 2013, A\&A, 558, A138

Boivin, L. P., 1978, Appl. Opt., 17, 3323

Born, M., \& Wolf, E. 2006, Principles of Optics, 7th edn. (Cambridge University Press)

Bout, M., Lamy, P., Maucherat, A., Colin, C., \& Llebaria, A. 2000, Appl. Opt., 39,22

Cady, E. 2012, Opt. Expr., 20, 15196
Cash, W. 2006, Nature, 442, 51

Evans, J. W. 1948, J. Opt. Soc. Am., 88, 1083

Fort, B., Morel, C., \& Spaak, G. 1978, A\&A, 63, 243

Goodman, J. W. 2005, Introduction to Fourier Optics (Roberts and Company Publishers)

Hamme, V. 1993, AJ, 106, 5

Jacquinot, P., \& Roizen-Dossier, B. 1964, Prog. Opt., 3, 31

Koutchmy, S. 1988, Sci. Rev., 47, 95

Landini, F., et al. 2016, Opt. Lett., 41, 757

Lemoine, D. 1994, J. Chem. Phys., 101, 3936

Lamy, P., Damé, L., Vivès, S., \& Zukhov, A. 2010, SPIE, 7731, 18

Lyot, B. 1939, MNRAS, 99, 580

Mas, D., Garcia, J., Ferreira, C., Bernardo, L. M., \& Marinho, F. 1999, Opt. Commun., 164, 233

Newkirk Jr., G., \& Bohlin, D. 1965, A\&A, 28, 234

Purcell, J. D., \& Koomen, M. J. 1962, in Report of NRL Progress (Washington, DC: US GPO), 9

Renotte, E., Alia, A., Bemporad, A., 2015, SPIE, 9604, 96040A

Rougeot, R., Flamary, F., Galano, D., \& Aime, C. 2017, A\&A, 590, A2

Vanderbei, R. J., Spergel, D. N., \& Kasdin, N. J. 2003, ApJ, 590, 593

Vanderbei, R. J., Kasdin, N. J., \& Cady, E. 2007, ApJ, 665, 794

Verroi, E., Frassetto, F., \& Naletto, G. 2008, J. Opt. Soc. Am. A, 25, 1

Wolfram 2012, Mathematica (Champaign, IL: Wolfram Research, Inc.) 


\section{Appendix A: Deviation of the sampling condition for the FFT technique}

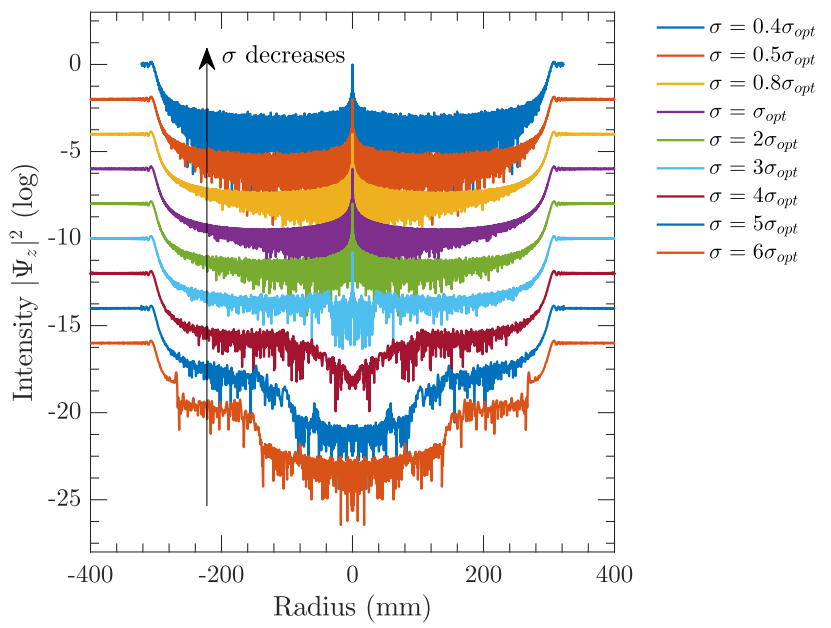

Fig. A.1. Horizontal cuts of the diffraction intensity $\left|\Psi_{z}(x, y)\right|^{2}$ in logarithmic scale, computed with the 2D FFT technique, for a fixed size $K=32768$ and for various samplings $\sigma$. The occulter is a sharp-edged disk of radius $R=300 \mathrm{~mm}$. The curves are shifted by two decades with respect to each other. From bottom to top: $\sigma=295.3 \mu \mathrm{m}, 246.1 \mu \mathrm{m}$, $196.9 \mu \mathrm{m}, 147.7 \mu \mathrm{m}, 98.4 \mu \mathrm{m}, \sigma_{o p t}=49.2 \mu \mathrm{m}, 39.4 \mu \mathrm{m}, 24.6 \mu \mathrm{m}$, and $19.7 \mu \mathrm{m}$.

In this appendix we explore the deviation from the sampling rules we established in Sect. 2. Here, we take as study case a sharp-edged disk with a radius $R=300 \mathrm{~mm}$. We fixed the size of the array to $K=2^{15}=32768$. Equations (10) and (6) give the relations constraining the best-fit sampling $\sigma_{o p t}$,

$\sigma_{o p t}=\sqrt{\frac{\lambda z}{K}}=49.2 \mu \mathrm{m}$

$\sigma_{o p t}<\frac{\lambda z}{2 R}=55.9 \mu \mathrm{m}$

$\sigma_{o p t}>\frac{2 R}{K}=43.3 \mu \mathrm{m}$

Figure A.1 shows a cut of the diffraction intensity $\left|\Psi_{z}(x, y)\right|^{2}$ in logarithmic scale for various samplings: $0.4,0.5,0.8,1,2,3$, 4,5 , and 6 times the value $\sigma_{\text {opt }}$. The curves are shifted by two decades in the vertical axis with respect to each other.

First, when $\sigma<\sigma_{\mathrm{opt}}$, the Fresnel filter is no longer correctly sampled because the variation of its phase at the edge of the array exceeds $\pi$. Related curves are therefore much more noisy. We also note that the spatial field decreases accordingly, and Eq. (A.3) is no longer fulfilled. Second, when $\sigma>\sigma_{\text {opt }}$, Eq. (A.2) is not respected. The structure of the Arago spot even disappears quickly as the sampling increases with respect to the nominal value because the required high spatial frequencies to described the disk are not captured. In the end, the best diffraction pattern is correctly obtained for $\sigma_{\text {opt }}$.

\section{Appendix B: Detailed expression of the Rubinowicz boundary integral}

We recall the general expression of the diffraction disturbance $\Psi_{z}^{(d)}$ given in Eq. (14):

$\Psi_{z}^{(d)}(x, y)=\frac{1}{4 \pi} \int_{\partial \mathcal{A}} \frac{\exp \left(\frac{2 i \pi}{\lambda}(s-z)\right)}{s-z} \times \frac{-(\xi-x) l_{\eta}+(\eta-y) l_{\xi}}{s} \mathrm{~d} l$

where $\boldsymbol{l}=\left(\boldsymbol{l}_{\xi}, \boldsymbol{l}_{\eta}, \mathbf{0}\right)$ is the unit vector in the direction tangent to the edge at any point $P=(\xi, \eta, 0)$ of $\partial \mathcal{A}, s$ is the distance between $P$ and the observation point $(x, y, z)$, and $\mathrm{d} l$ is the differential curvilinear element of the edge.

In the case of the disk, we take advantage of the axisymmetry, and the function $\Psi^{(d)}$ is written as a radial function. The edge can be parametrized by its polar angle $\phi$, such that $\xi=R \cos \phi$ and $\eta=R \sin \phi$. The unit tangent vector, counted counterclockwise, at any point $P(\phi)$ is simply $l=(\sin \phi,-\cos \phi, 0)$. Thus, the diffraction disturbance becomes

$$
\begin{gathered}
\Psi_{z}^{(d)}(r)=\frac{1}{4 \pi} \int_{0}^{2 \pi} \frac{\exp \left(\frac{2 i \pi s(\phi)}{\lambda}\right)}{s(\phi)-z} \times \frac{R-r \cos \phi}{s(\phi)} R \mathrm{~d} \phi \\
\text { with } \quad s(\phi)=\sqrt{R^{2}+r^{2}-2 R r \cos \phi+z^{2}}
\end{gathered}
$$

The same expansion can be performed for a serrated occulter. We recall that the occulter is defined by its number of teeth $N_{t}$ and the tooth height $\Delta$. Two other important geometrical parameters are the half-apex angle $\Lambda$ of the teeth, and the half angular separation $\hat{N}_{1 / 2}$ of two successive teeth, as given in Eq. (17). In polar coordinates, the edge of the serrated occulter is given by

$\rho(\phi)=\frac{R+\Delta}{\cos \phi^{\prime}+\cot \Lambda \times\left|\sin \phi^{\prime}\right|}$,

with : $\phi^{\prime}=\bmod \left(\phi, 2 \hat{N}_{1 / 2}\right)-\hat{N}_{1 / 2}$

where $\bmod (a, b)$ is the rest of the Euclidean division of $a$ by $b$. Then, by differentiating the expressions of $\xi=\rho \cos \phi$ and $\eta=\rho \sin \phi$, we obtain the two components $l_{\xi}, l_{\eta}$ of the tangent vectors,

$l_{\xi}(\phi)=\frac{\sin \left(\phi-\phi^{\prime}\right)-\operatorname{sign}\left(\phi^{\prime}\right) \times \cot \Lambda \times \cos \left(\phi-\phi^{\prime}\right)}{\sqrt{1-\cot ^{2} \Lambda}}$
$l_{\eta}(\phi)=\frac{\cos \left(\phi-\phi^{\prime}\right)+\operatorname{sign}\left(\phi^{\prime}\right) \times \cot \Lambda \times \sin \left(\phi-\phi^{\prime}\right)}{\sqrt{1-\cot ^{2} \Lambda}}$,

where $\operatorname{sign}(a)$ is the function returning the sign of $a$. Injecting Eqs. (B.4), (B.5) into Eq. (B.1), we derive the analytical integral over $\phi$ that gives the diffraction disturbance $\Psi_{z}^{(d)}$. This integral may be numerically solved using dedicated integration tools. 
R. Rougeot and C. Aime: Theoretical performance of serrated external occulters for solar coronagraphy

\section{Appendix C: Variation of diffraction intensity with wavelength}

In this section, we illustrate the variation in diffraction intensity $\left|\Psi_{z}\right|^{2}$ with wavelength. Here, the occulter is made of $N_{t}=128$ teeth of $\Delta=20 \mathrm{~mm}$. We consider different wavelengths $\lambda$ in white light, the bandpass of ASPIICS. Figure C.1 shows some results to illustrate this: a cut of the diffraction intensity $\left|\Psi_{z}(x, y)\right|^{2}$ in logarithmic scale for $\lambda=540,550,560$, and $570 \mathrm{~nm}$ (a)

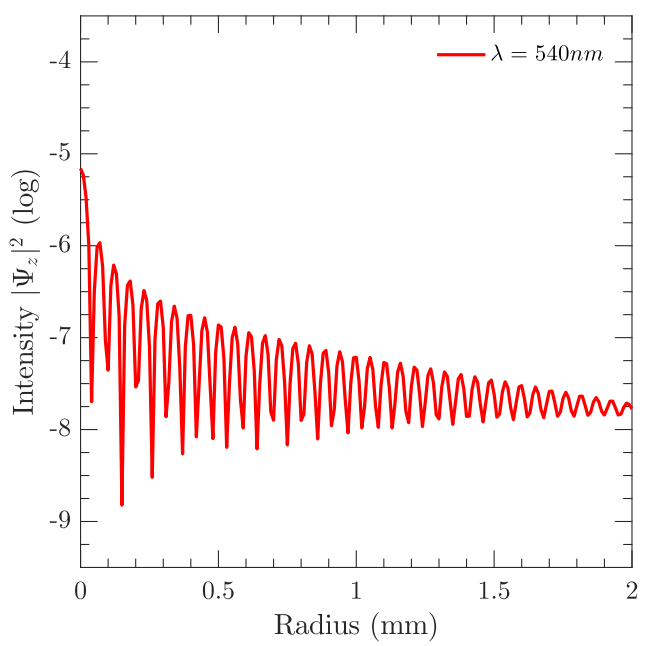

(c)

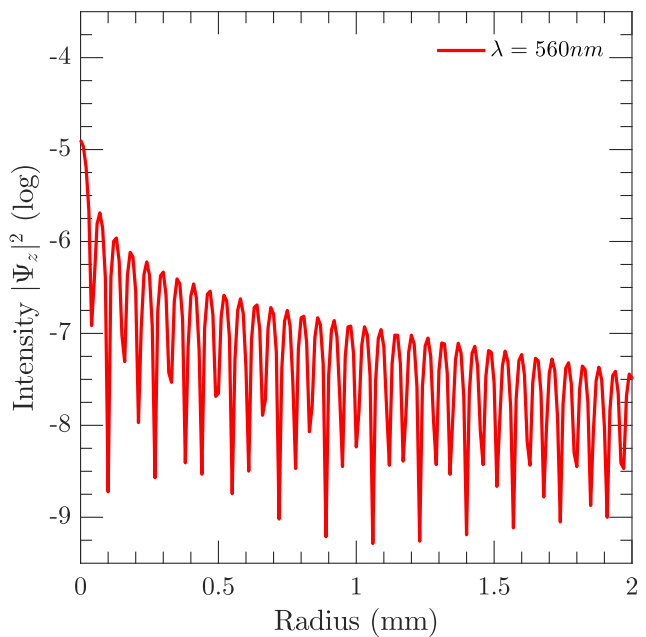

(b)

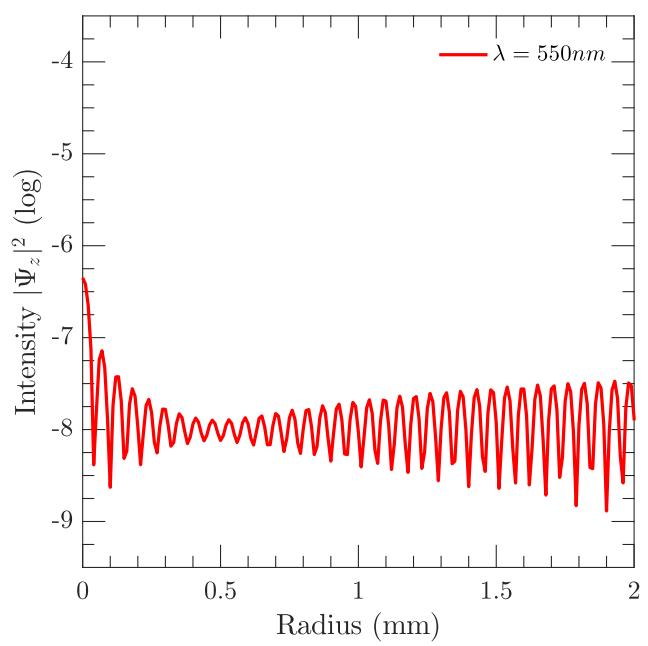

(d)

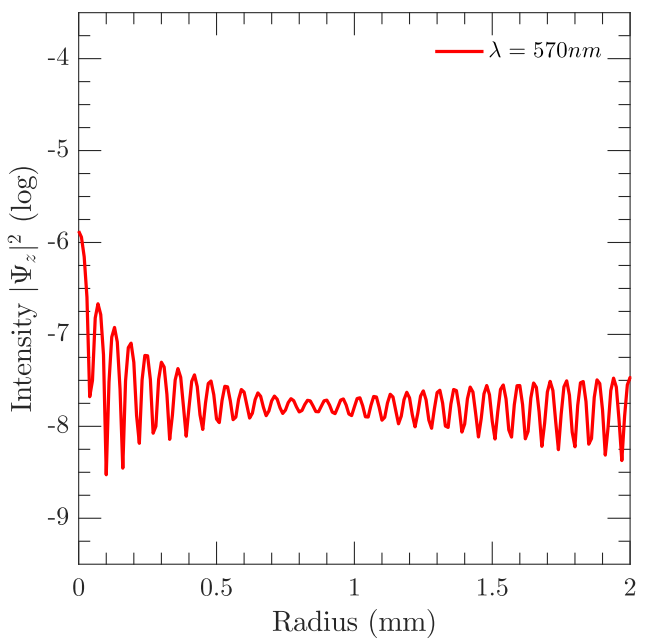

Fig. C.1. Horizontal cuts near the center of the diffraction intensity $\left|\Psi_{z}(x, y)\right|^{2}$ in logarithmic scale. The occulter is made of $N_{t}=128$ teeth of $\Delta=20 \mathrm{~mm}$. Panel $a: \lambda=540 \mathrm{~nm}$. Panel $b: \lambda=550 \mathrm{~nm}$. Panel $c: \lambda=560 \mathrm{~nm}$. Panel $d: \lambda=570 \mathrm{~nm}$. 